\title{
Chronic Cutaneous Hyalohyphomycosis by Paecilomyces
}

\author{
N. Boufflette ${ }^{1}$, J.E. Arrese ${ }^{2}$, P. Leonard ${ }^{3}$ and A.F. Nikkels ${ }^{*}, 1$ \\ Departments of ${ }^{1}$ Dermatology, ${ }^{2}$ Dermatopathology and ${ }^{3}$ Infections Diseases, University Hospital of Liège, Liège, \\ Belgium
}

\begin{abstract}
Paecilomyces lilacinus is a ubiquitous saprophytic fungus that rarely causes infections in humans, frequently affecting the eyes and the skin. Cutaneous and subcutaneous infections mainly occur in immunocompromised hosts but have occasionally been reported in immunocompetent patients. The clinical spectrum is highly heterogeneous and diagnosis is often delayed.
\end{abstract}

A 60-year-old woman with idiopathic chronic necrotizing vasculitis treated since 10 years with a series of immunosuppressive therapies presented since three years various clinical presentations of chronic hyalohyphomycosis caused by $P$. lilacinus. Diagnosis was only obtained three years after the first clinical signs, following the histologic analysis of the surgical excision of a cutaneous abscess. Treatment with oral voriconazole was successful.

This case report illustrates the highly heterogeneous clinical aspects of hyalohyphomycosis by P. lilacinus leading to a delay in diagnosis and treatment, particularly in the immunosuppressed patient.

Keywords: Hyalohyphomycosis, immunosuppression, paecilomyces lilacinus, voriconazole, skin infection.

\section{INTRODUCTION}

Hyalohyphomycosis is a rare infection caused by fungi that produce hyaline septate hyphae in tissues [1]. Etiological agents include, among others, species of Acremonium, Fusarium, Scopulariopsis, Paecilomyces and Beauveria. The genus Paecilomyces was identified for the first time in 1907 [2]. P. lilacinus has only been assigned since 1974 to the genus Paecilomyces [3]. The lilac-colored colony and the production of a deep purplish-red pigment on Czapek solution agar are characteristic. P. lilacinus and $P$. variotii are the two major species, which are most frequently associated with infections in human. Most of these occur in immunocompromised hosts, while the incidence in immunocompetent hosts is also increasing [4]. Paecilomyces species are rare but emerging causes of hyalohyphomycosis. These fungi are regularly isolated from soil and air, decaying plants and food products [1]. Their potential resistance to sterilization techniques increases their clinical significance. Paecilomyces infections usually concern oculomycosis as well as cutaneous and subcutaneous infections [1], and occasionally sinusitis, fungaemia, onychomycosis, lung abscess, pleural effusion, osteomyelitis, peritonitis and endocarditis.

Cutaneous and subcutaneous infections have been reported to be highly heterogeneous and may be notoriously difficult to diagnose. Herein, the polymorphous clinical and histologic features of chronic cutaneous hyalohyphomycosis related to $P$. lilacinus are illustrated in a patient with

*Address correspondence to this author at the Department of Dermatology, University Hospital of Liège, Liège, Belgium; Tel: +32 4366 7232;

Fax: +32 4366 7234; E-mail: af.nikkels@chu.ulg.ac.be longstanding immunosuppressive therapies for idiopathic necrotizing vasculitis.

\section{CASE REPORT}

A 60-year-old woman presented a tender and asymptomatic swelling, $5 \mathrm{~cm}$ in diameter, at the posterior aspect of her left arm (Fig. 1). According to the patient, she had this swelling for over 12 months. The lesion appeared progressively and increased in size over time. The lesion was mistaken clinically as a lipoma and echographically as a hematic collection and no treatment was initiated. The patient also noticed the development of three subcutaneous nodular and pustular lesions in the immediate vicinity of the swelling during the last few weeks (Fig. 2). Furthermore, she complained of an erythematous-violaceous irregularly shaped infiltrated large plaque of the entire anterolateral aspect of her left thigh (Fig. 3). This lesion was also present since more than three years and several punch and excisional biopsies were performed without however evidencing the presence of fungal agents despite periodic acid-Schiff (PAS) and Gomori-Grocott histochemical stainings. She denied any trauma related to these areas and had no systemic complaints. She was a febrile and without palpable regional lymph nodes.

The patient had been followed for over 10 years in the rheumatology department for anecrotizing vasculitis. Extensive and repetitive workups remained negative (antinuclear antibodies, $\mathrm{p}$ and cANCA's cryoglobulines, anti-phospholipids, etc) and the vasculitis was classified as idiopathic. Over time, she received several lines of immunosuppressive therapies: cyclophosphamide, rituximab IV (anti-CD20 monoclonal antibodies), azathioprine and systemic corticosteroids. The cutaneous lesions of her idiopathic necrotizing vasculitis occurred most of the times 
on her legs. Every time noticing a new lesion in the lower extremities, the rheumatologist suspected a recurrence of vasculitis and treated the patient with systemic corticosteroids. Current medication included methylprednisolone 12 $\mathrm{mg}$ daily, azathioprine 50mg tid, esomeprazol $40 \mathrm{mg}$ daily, calcium carbonate $1250 \mathrm{mg}$ daily and vitamin D $100.000 \mathrm{UI}$ weekly. On physical examination, blood pressure was $140 / 90 \mathrm{mmHg}$, respiratory rate was 18 breaths/min and pulse rate $100 / \mathrm{min}$ with a regular rhythm. All laboratory work including a complete blood count, chemistry and liver panel was in normal range.

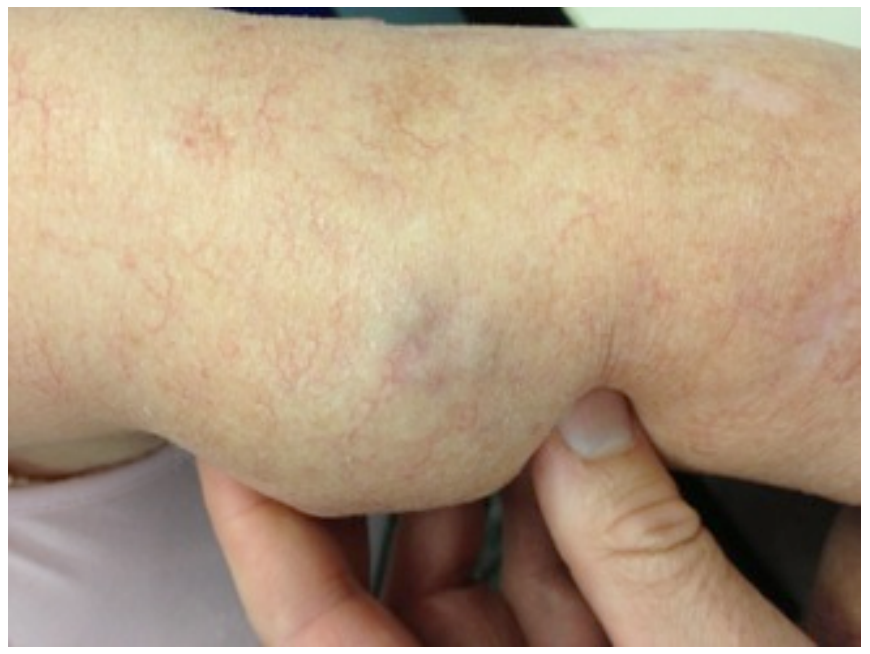

Fig. (1). Cutaneous Paecilomyces lilacinus infection onthe left arm characterized bya large and tender swelling.

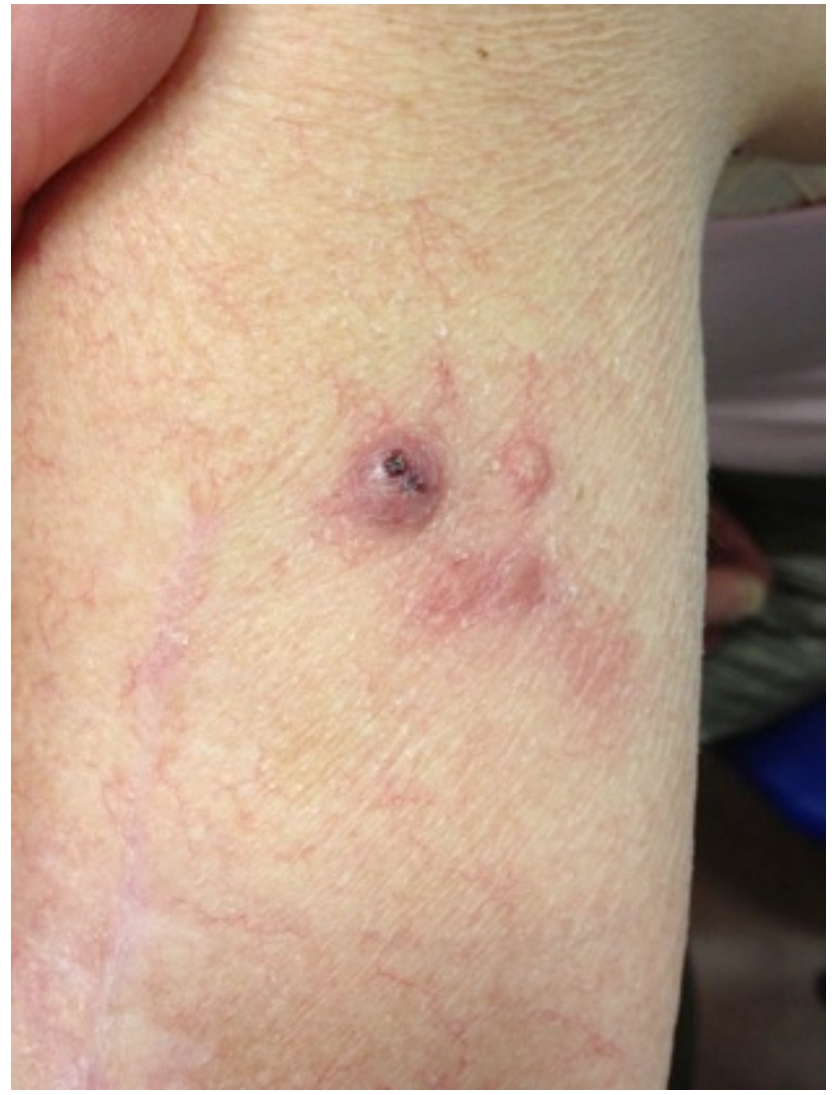

Fig. (2). Cutaneous nodular lesions by Paecilomyces lilacinus on the left arm.

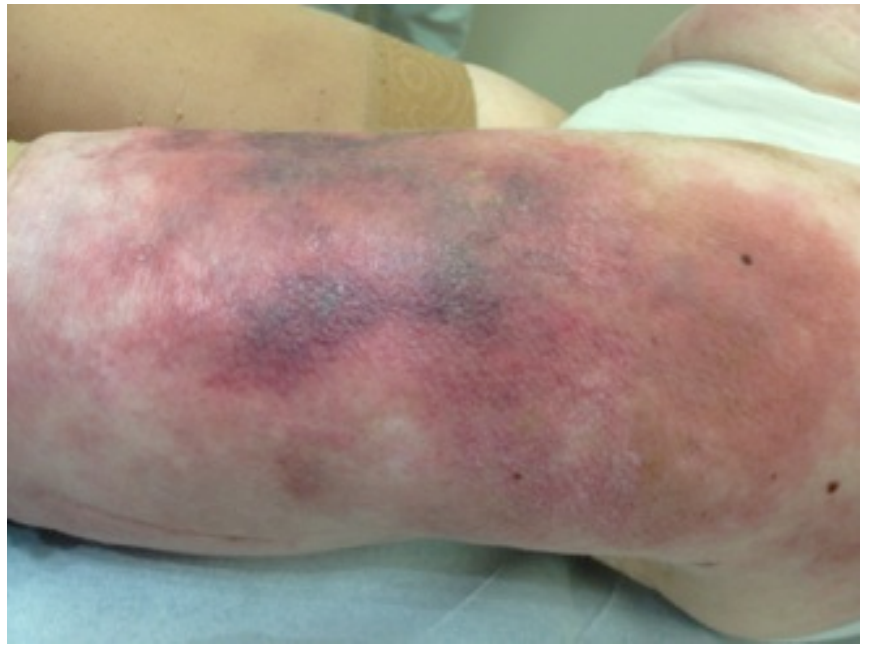

Fig. (3). Chronic cutaneous Paecilomyces lilacinus infection on left thigh characterized byan erythematous-violaceous, partially necrotic and infiltrated plaque.

Due to the longstanding administration of azathioprine and her fair phototype, the patient had already presented several squamous cell carcinomas (SCC). She presented with a clinical suspicion of three new SCC's on her left upper arm. Surgical excision was decided and simultaneously she requested the excision of the large swelling on the anterior aspect of her left upper arm. On excision, a large amount of pustular secretions was evidenced requiring the insertion of a drain. Histology of the biopsy specimens revealed a heavily necrotic and granulomatous reaction with giant cells and few inflammatory cells in the dermis on haematoxylin/eosin staining (Fig. 4). PAS (Fig. 5) and Gomori-Grocott methenamine-silver (Fig. 6) histochemical stainings noted the presence of hyaline hyphae within the dermal necrotic tissue with numerous septate hyphae associated with the presence of spores. Giemsa staining was negative. The final histologic result was a hyalohyphomycosis. Bacterial cultures of the fluid were negative. Fungal culture, however, grew a mold, which was subsequently identified as $P$. lilacinus after a subculture on an enriched medium. Fungal cultures of blood presented no growth. Although no

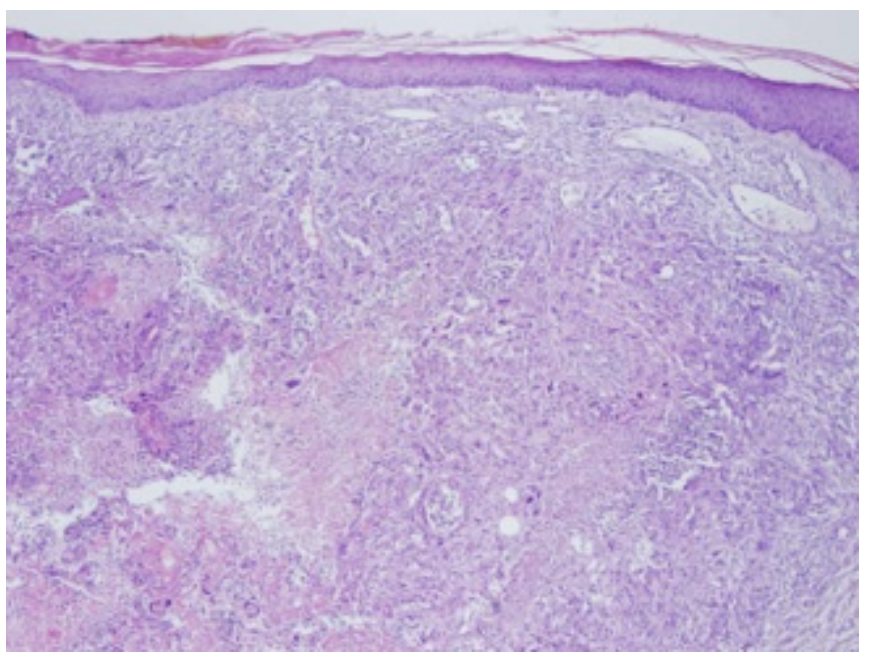

Fig. (4). Granulomatous and necrotic infiltration of the entire reticular dermis $(\mathrm{H} / \mathrm{E}, \mathrm{x} 4)$. 
breakpoints have been established for molds, antifungal susceptibility showed an elevated minimum inhibitory concentration (MIC) for amphotericin B (MIC $>32 \mu \mathrm{g} / \mathrm{ml}$ ) and itraconazole (MIC $>32 \mu \mathrm{g} / \mathrm{ml}$ ). However, a low MIC for voriconazole (MIC $=0,094 \mu \mathrm{g} / \mathrm{ml}$ ) was noted. Therefore, a treatment with oral voriconazole was initiated. The loading dose was $300 \mathrm{mg}$ bid on the first day, followed by $200 \mathrm{mg}$ bid, prescribed for a period of 3 months. During the first 4 weeks of treatment, clinical resolution progressed nicely in the left thigh and the scar of the surgery performed in the left arm was healthy. The patient suffered no major side effects related to the treatment except for a slight elevation of liver enzymes. Unfortunately, the patient died during the fifth week of treatment from a bacterial pneumonia.

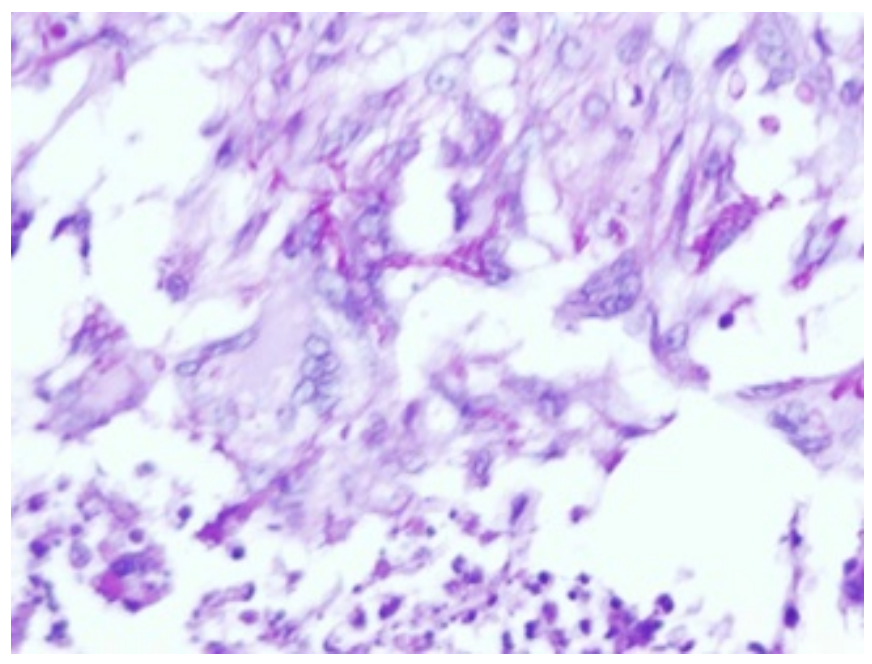

Fig. (5). Demonstration of septate hyphae in red (PAS, x40).

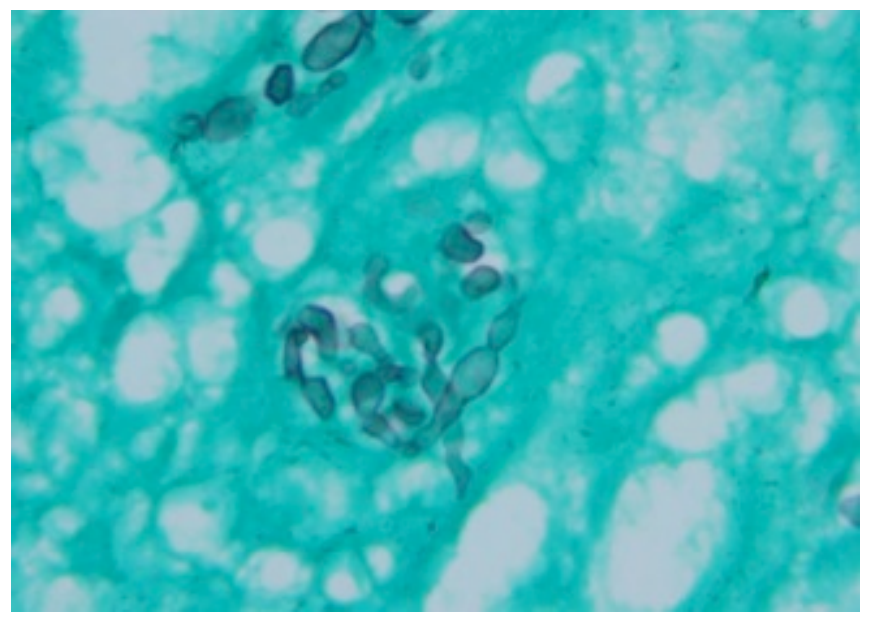

Fig. (6). Grocott-Gomori methenamine-silver staining of a biopsy specimen showing regular filamentous fungi and spores.

\section{DISCUSSION}

In most of the infections caused by these environmental fungi, some predisposing factors were found. As to cutaneous and subcutaneous $P$. lilacinus infections, risk factors were mainly solid organ and bone marrow transplantation, malignancies, corticosteroid therapy, primary immunodeficiency, diabetes mellitus and AIDS [1]. Our patient presented a chronic and severe iatrogenic immunosuppression following several immunosuppressive regimens used for over 10 years to treat her necrotizing vasculitis. The portal of entry attributed to this fungus usually involves a breakdown of the skin barrier, in dwelling catheters or inhalation. There was no evidence of hematogenous spread in our patient and fungal blood cultures were negative. We hypothesized that our patient was contaminated by direct inoculation through an unnoticed, minor skin abrasion, facilitated by a highly atrophic skin induced by chronic systemic corticosteroids [5].

Cutaneous and subcutaneous infections present numerous clinical manifestations such as solitary or disseminated skin eruptions with erythematous macules, papules, vesicles, or nodules with a necrotic center [1]. Our patient presented several clinical presentations such as nodular lesions and a swelling of the arm, as well as a large, irregular, infiltrated and indurated plaque on the thigh. As illustrated in our case, these highly polymorphous clinical images frequently lead to a delay in diagnosis subsequently interfering with adequate treatment.

Fungal culture and histology of the lesions are required to diagnose a $P$. lilacinus infection. Even if histological examination can identify fungal structures, the fungal culture remains the gold standard for fungal identification. Paecilomyces molds grow fast on Sabouraud dextrose agar and the pathogen can be identified within one to two weeks [6].

Because of their different antifungal susceptibilities, the differentiation between Paecilomyces species is mandatory to treat the disease correctly. P. lilacinus has a high resistnce to conventional antifungal drugs unlike $P$. variotii, which has a greater susceptibility to these agents [7]. Given $P$. lilacinus' broad resistance, it is always useful to realize a susceptibility test in order to define the most appropriate treatment. In vitro studies of $P$. lilacinus antifungal susceptibility have shown poor activity of older antifungal drugs (amphotericin B, flucytosine and fluconazole) and contradiction exists concerning data regarding the activity of the first-generation azoles, such as ketoconazole, miconazole, clotrimazole and itraconazole [7]. The secondgeneration triazoles, such as voriconazole, posaconazole and ravuconazole present a good antifungal effect [8]. More specifically, the last two drugs show very low MICs although no data have been published concerning their clinical use. Currently, there is no standard treatment regimen for $P$. lilacinus infections. Although the clinical experience with voriconazole is limited, it is recommended as first-line therapy [9]. Up to date, only seven published cases of skin infection treated with voriconazole, which were all successful. Although we were unable to follow the entire evolution of the lesions, a spectacular regression was observed during the first 4 weeks with voriconazole treatment.

Treatment duration with voriconazole remains undetermined, ranging from 3 weeks to 10 months [10,11]. The treatment duration should rely on the clinical resolution of the infection and/or the presence of adverse effects linked to the medication. The most common adverse effects of voriconazole included visual disturbances, increased levels of hepatic enzymes and skin rash through photosensitization [12]. Surgical excision should also be considered on a caseby-case basis. 
In conclusion, this case illustrates the highly polymorphous spectrum of $P$. lilacinus skin infection. Performing susceptibility tests is mandatory as resistance is not uncommon. Surgery should not be overlooked as treatment option.

\section{AUTHORS' CONTRIBUTIONS}

All authors provided substantial contributions to the conception and design, acquisition of data, or analysis and interpretation of data, to the drafting of the article or revising it critically for important intellectual content. All authors provided final approval of the version to be published.

\section{CONFLICT OF INTEREST}

The authors confirm that this article content has no conflict of interest.

\section{ACKNOWLEDGEMENTS}

Declared none.

\section{REFERENCES}

[1] Pastor FJ, Guarro J. Clinical manifestations, treatment and outcome of Paecilomyces lilacinus infections. Clin Microbiol Infect. 2006; 12: 948-60.

[2] Bainer G. Mycothèque de l'école de pharmacie XI. Paecilomyces, genre nouveau de mucédinées. Bull Soc Mycol Fr 1907; 23: 26-7.

[3] Samson RA. Paecilomyces and some allied hyphomycetes. Stud Mycol 1974; 6: 58-62.
[4] Carey J, D’Amico R, Sutton DA, Rinaldi MG: Paecilomyces lilacinus vaginitis in an immunocompetent patient. Emerg Infect Dis 2003; 9: 1155-8.

[5] Itin PH, Frei R, Lautenschlager S, et al. Cutaneous manifestations of Paecilomyces lilacinusinfection induced by a contaminated skin lotion in patients who are severely immunosuppressed. J Am AcadDermatol1998; 39: 401-9.

[6] Hall VC, Goyal S, Davis MD, Walsh JS. Cutaneous hyalohyphomycosis caused by Paecilomyces lilacinus: report of three cases and review of the literature. Int J Dermatol 2004; 43: 648-53.

[7] Aguilar C, Pujol I, Sala J, Guarro J. Antifungal susceptibilities of Paecilomyces species. Antimicrob Agents Chemother 1998; 42: 1601-4.

[8] Castelli MV, Alastruey-Izquierdo A, Cuesta I, et al. Susceptibility testing and molecular classification of Paecilomyces spp Antimicrob Agents Chemother 2008; 52: 2926-8.

[9] Rimawi RH, Carter Y, Ware T, Christie J, Siraj D. Use of voriconazole for the treatment of Paecilomyces lilacinuscutaneous infections: case presentation and review of published literature. Mycopathologia 2013; 175: 345-9

[10] Hilmarsdottir I, Thorsteinsson SB, Asmundsson P, Bodvarsson M, Arnadottir M: Cutaneous infection caused by Paecilomyces lilacinusin a renal transplant patient: treatment with voriconazole. Scand J Infect Dis 2000; 32: 331-2.

[11] Martin CA, Roberts S, Greenberg RN. Voriconazole treatment of disseminated Paecilomyces infection in a patient with acquired immunodeficiency syndrome. Clin Infect Dis 2002; 35: e78-81.

[12] Sabo JA, Abdel-Rahman SM: Voriconazole: a new triazole antifungal. Ann Pharmacother 2000; 34: 1032-43.

(C) Boufflette et al.; Licensee Bentham Open.

This is an open access article licensed under the terms of the Creative Commons Attribution Non-Commercial License (http://creativecommons.org/licenses/ by-nc/3.0/) which permits unrestricted, non-commercial use, distribution and reproduction in any medium, provided the work is properly cited. 